\title{
Shining a light on balance and hearing
}

Oyster toadfish (Opsanus tau) are commonly used in research on vestibular physiology, the study of balance. Recent research has examined hair cells from the toadfish's crista ampullaris, a structure in the inner ear that senses and responds to movement of the head. The hair cells convert mechanical vibrations produced by sound, gravity or motion into signals that are transmitted to the brain by adjacent nerve cells.

The new research showed that hair cells could be stimulated by pulses of infrared radiation to send signals to the brain. This type of optical, rather than electrical, stimulation had been previously shown to activate nerve and heart cells in various animal models. In a press release, Richard Rabbitt (University of Utah, Salt Lake City), who led the research, summarized, "[We] talk to the brain with optical infrared pulses instead of electrical pulses." The results have potential therapeutic applications for hearing loss and for certain balance and movement disorders (such as Parkinson's disease) in humans.

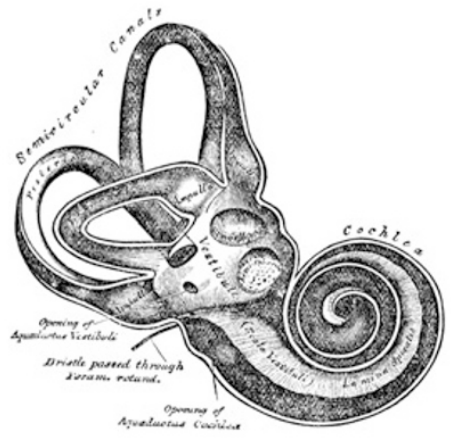

cycle is a normal cellular process, but "it's normally controlled by the cell, not by us," said Rabbitt in a press release, continuing, "the infrared radiation gives us a tool to control the cell."

These results may someday lead to the development of improved cochlear implants to treat deafness. Currently available cochlear implants convert sound into electrical signals that are typically transmitted to a number of electrodes (8-24). The number of electrodes is limited, and these implants provide a limited range of hearing. Optical stimulation, instead of electric, may enable a wider range of sound sensitivity, more realistically representing natural hearing. Such implants would probably not be available for at least 5-10 years, according to Rabbitt.

Similarly, optical stimulation may also enable the development of devices to preserve vision in certain types of vision loss and to maintain balance and restore movement in disorders like Parkinson's disease. Monica Harrington

\section{OVERCOMING IMMUNOSUPPRESSTVE TUMORS}

For patients diagnosed with pancreatic ductal adenocarcinoma who are not candidates for surgery, chemotherapy offers little benefit compared to best supportive care. Now, a clinical trial in humans and a study in mice have identified a new way to break down the defenses of this deadly cancer, offering hope for new potential treatments (Science 331, 1612-1616; 2011).

Pancreatic ductal adenocarcinoma forms a tough stroma, or shell, around itself, making it difficult for drugs to reach tumor cells. Additionally, the white blood cells become entwined with tumor cells, leading to suppression of the immune system. To try to develop an effective treatment regimen, Robert Vonderheide of the University of Pennsylvania School of Medicine in Philadelphia and colleagues set out to reverse this immune suppression. They hypothesized that activating a protein called CD40 that is known to be involved in the activation of immune cells would lead to the activation of antitumor T cells.

The research team administered this antibody therapy to 21 patients with inoperable pancreatic ductal adenocarcinoma who were receiving standard chemotherapy treatment. These patients survived, on average, for several weeks longer than would have been expected with chemotherapy treatment alone. The team observed tumor regressions in some of these patients. When the researchers biopsied two of the shrunken tumors, they found no T cells but did find macrophages, another type of immune cell.

To understand the mechanism underlying the efficacy of this therapy, the researchers tested the antibody treatment in mice with a genetically engineered model of pancreatic ductal adenocarcinoma. They found that $30 \%$ of mice treated with a similar antibody therapy had tumor regression, regardless of whether the mice also received chemotherapy. Further analyses showed that the antibody caused T cells to become activated but that the T cells did not move from the lymph nodes to the tumors. The macrophages, however, did move into the tumors and also helped break down the shell surrounding the tumors. When the researchers depleted macrophages from the mice with cancer, the antibody treatment failed to induce degradation of the stroma, showing that macrophages are required for this treatment to be effective.

This study shows that immune-based cancer treatments do not necessarily need to rely on inducing T cell activity; this particular treatment regimen relied on macrophages instead of T cells. The researchers conclude that their findings "support the continued development of emerging therapeutic strategies that target inflammatory cells and stroma within the tumor microenvironment."

Kirsten Dorans 